\title{
La elaboración del índice de precios al consumidor (IPC) ante la emergencia del COVID-19 en América Latina y el Caribe
}

\section{Resumen}

En el contexto de la pandemia mundial de la enfermedad por coronavirus (COVID-19), la Comisión Económica para América Latina y el Caribe (CEPAL) está elaborando una serie de publicaciones cortas con recomendaciones de política relevantes para este período. En la presente nota se plantean una serie de recomendaciones para la continuidad de la recopilación de la información de precios con la que se calcula el índice de precios al consumidor (IPC).

\section{Introducción}

En los países de América Latina y el Caribe, y con el objetivo de contener la propagación del COVID-19, muchos Gobiernos han tomado la decisión de imponer restricciones a la movilidad de las personas con medidas de cuarentena. Esto ha llevado a interrumpir operaciones estadísticas en que la recolección de la información se realiza en forma presencial sobre el terreno. Es así como las instituciones que elaboran las estadísticas oficiales deben enfrentar desafíos complejos para seguir ofreciendo a sus autoridades y usuarios estadísticas oportunas y de calidad, entre ellas el IPC.

Teniendo en cuenta esta situación, la División de Estadísticas de la CEPAL dirigió un breve cuestionario a las oficinas nacionales de estadística y bancos centrales de América Latina y el Caribe para conocer los efectos de la emergencia sanitaria en el funcionamiento de las instituciones estadísticas. Las respuestas obtenidas de 20 países indican que, al 20 de marzo, todos los países pasaban por algún tipo de emergencia de salud debido al COVID-19.

Desde el 24 de marzo se han venido realizando una serie de videoconferencias para evaluar los impactos de la pandemia del COVID-19 sobre las operaciones estadísticas ${ }^{1}$. Estas videoconferencias han sido convocadas por la CEPAL, en conjunto con la Organización Internacional del Trabajo (OIT) y el Instituto Nacional de Estadísticas (INE) de Chile en su calidad de coordinador de las conversaciones virtuales de la Red de Transmisión del Conocimiento (RTC) de la Conferencia Estadística de las Américas de la CEPAL, que es presidida por Colombia a través del Departamento Administrativo Nacional de Estadística (DANE)

Asimismo, se realizaron teleconferencias y consultas puntuales con los países de la región de modo de conocer los avances realizados durante la primera quincena de marzo y posteriormente una vez terminado el mes

El objetivo de este documento es brindar información acerca del estado de situación en la región, así como dar a conocer algunas de las recomendaciones acordadas con los países para afrontar la coyuntura del COVID-19 en relación con la compilación de datos para el IPC. Si bien el análisis se aplica a este indicador, las reflexiones pueden extenderse a la compilación del sistema de precios en general, en la medida que sean aplicables. Las recomendaciones acordadas se apoyan en los manuales internacionales y en las pautas que los propios países ya han desarrollado en situaciones de desastres provocados por fenómenos naturales o sociales a lo largo de su historia.

\footnotetext{
Véase [en línea] https://rtc-cea.cepal.org/es/conectados-rtc/planes-de-contigencia-adoptados-por-lasoficinasnacionales-de-estadistica-ante-la
}
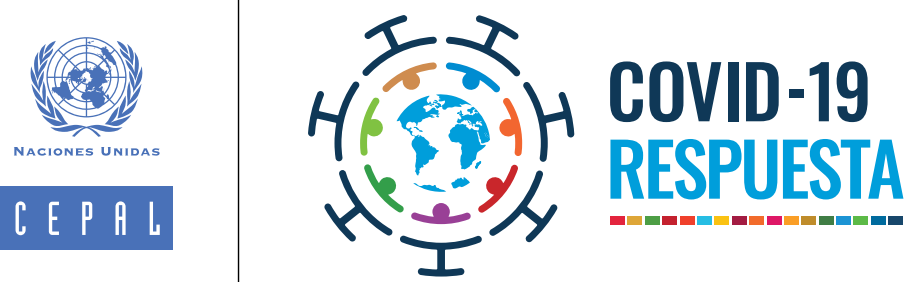

\section{Resumen}

Introducción

A. Levantamiento de precios y procesamiento de los datos para el IPC en marzo de 2020

$B$. Métodos de imputación de acuerdo con los criterios metodológicos internacionales y proceso de cálculo para el IPC

C. Desafíos observados en la implementación de las modalidades de recolección alternativas

D. Difusión de las cifras en el nuevo escenario

E. Recomendaciones

F. Aspectos de representatividad del indicador

Anexo 1 


\section{A. Levantamiento de precios y procesamiento de los datos para el IPC en marzo de 2020}

A mediados de marzo, los Gobiernos de todos los países de la región habían adoptado medidas de restricción de la movilidad de las personas mediante la declaración de la emergencia sanitaria y la cuarentena, por lo que las instituciones de estadística tuvieron que implementar modalidades alternativas para la recolección de la información y, en el caso del IPC, de la información sobre los precios de los bienes y servicios.

Sobre la base de la encuesta realizada a los países se identificaron las siguientes situaciones:

- Se alcanzó una recolección de precios que varía entre un 60\% y un 80\% para marzo de 2020

- Los precios no recolectados que corresponden a situaciones de estacionalidad o temporalidad (como, por ejemplo, verduras de otras temporadas que no están presentes en marzo o servicios de educación con comportamiento temporal) y que fueron cotizados en otros períodos contribuyen al porcentaje de precios no recolectados.

- La mayor parte de las instituciones de estadística alcanzaron a recolectar precios en las dos primeras semanas con la modalidad habitual, que corresponde a la forma presencial sobre el terreno, e incluso algunas alcanzaron a recolectar en la tercera semana del mes de marzo, antes de que se iniciara la cuarentena que restringe la movilidad de las personas.

- Los locales que brindan servicios de recreación y cultura, así como los restaurantes y hoteles fueron cerrados antes de concluir el mes en gran parte de los países de la región, por lo cual el levantamiento de los precios correspondientes no se pudo completar, lo que representa una cifra menor del 10\% del total de precios recolectados.

- El mes de marzo de 2020 tendrá un porcentaje de imputación superior al habitual (entre un 3\% y un 5\%) pues la recolección se vio afectada por la declaración de cuarentena en los países, medida que llevó al cierre de locales en algunos casos, problemas de movilidad de los encuestadores, así como una mayor demanda de ciertos bienes de primera necesidad, generando una baja en las existencias de manera temporal, entre otras dificultades. Se espera que los porcentajes de imputación por estos motivos alcancen entre un $10 \%$ y un $30 \%$ del total, aproximadamente.

Cuadro 1

Aspectos principales de la recolección de precios del IPC a marzo de 2020

\begin{tabular}{|l|l|l|}
\hline $\begin{array}{l}\text { Porcentaje de } \\
\text { precios recolectados }\end{array}$ & $\begin{array}{l}\text { Efectos de la situación } \\
\text { de emergencia sanitaria }\end{array}$ & $\begin{array}{l}\text { Rubros afectados por la situación } \\
\text { de emergencia sanitaria }\end{array}$ \\
\hline Entre el 60\% y el 80\% & $\begin{array}{l}\text { Restricción de movilidad para los } \\
\text { funcionarios de campo }\end{array}$ & $\begin{array}{l}\text { Todos aquellos rubros cuyos precios } \\
\text { se levantan de manera presencial }\end{array}$ \\
\hline Cierre de establecimientos & $\begin{array}{l}\text { Servicios de recreación: cines, teatros, } \\
\text { espectáculos deportivos, entre otros } \\
\text { Servicios de comida fuera de casa, } \\
\text { servicios de hoteles }\end{array}$ \\
\hline & Bienes sin disponibilidad temporal & $\begin{array}{l}\text { Bienes de cuidado personal } \\
\text { y de cuidado del hogar }\end{array}$ \\
\hline
\end{tabular}

Fuente: Comisión Económica para América Latina y el Caribe (CEPAL), sobre la base de información de los países.

Las nuevas condiciones representan un reto para la elaboración de estadísticas oficiales en cada país, especialmente en las operaciones estadísticas con investigación continua y presencial en los territorios, por ello debieron implementarse nuevas modalidades de recolección de precios, utilizando alternativas, en lo posible, con soporte en el uso de tecnologías de la información y las comunicaciones (TIC). Por lo tanto, en este nuevo escenario las instituciones de estadística optaron por implementar en sus operativos de recolección herramientas como llamadas telefónicas a sus informantes, correo electrónico, recolección vía Internet (investigación directa en las fuentes que cuentan con sitios web, redes sociales o en las páginas web para la compra en línea, entre otras), además de aplicar el uso de catálogos actualizados (impresos o digitales) y precios y características de los productos provistos por funcionarios de la institución como resultado de las compras personales. 
Estas nuevas modalidades han permitido, en algunas situaciones, mejorar la cobertura del levantamiento de precios y alcanzar un mayor número de bienes y servicios, llegando a cubrir entre un $10 \%$ y un $30 \%$ de los precios a levantarse. Finalmente, con estas nuevas herramientas, los países alcanzaron para la medición del IPC en marzo niveles adecuados (levantamiento de precios superior al 50\% del total de los precios recolectados) para realizar los cálculos del índice. La información que no se levantó debió imputarse según los métodos definidos en los respectivos algoritmos de cálculo de cada institución según consta en sus metodologías.

\section{B. Métodos de imputación de acuerdo con los criterios metodológicos internacionales y proceso de cálculo para el IPC}

En la situación de precios faltantes (no observados) el registro deberá imputarse según los criterios metodológicos del país, que deberían estar en línea con las recomendaciones internacionales ${ }^{2}$. Los países cuentan con una metodología de imputación según se trate de un producto estacional o no estacional utilizada sobre los precios faltantes con tasa de imputación habitual para un período mensual del 3\% al 7\%. Sin embargo, en el escenario actual, la tasa de precios faltantes resultará superior a la habitual y, por ello, el porcentaje de precios a ser imputados será mayor

Cabe señalar que los procedimientos de imputación buscan asignar una variación a un registro de precio faltante; para ello, las recomendaciones internacionales ofrecen diferentes alternativas según el tipo de producto y el nivel de desagregación. Las reglas de imputación se aplican en forma secuencial ascendente, desde el agregado elemental (variedad-establecimiento) hasta el nivel de agregación superior (producto). A continuación, se especifican los mecanismos de imputación tradicionales.

a) Precio faltante para una variedad especifica en un establecimiento individual: se imputa a este registro la variación media geométrica para la misma variedad especifica de los demás establecimientos que lo informan.

b) Precios faltantes para todos los establecimientos informantes de la misma variedad específica: se imputa para esa variedad y todos sus establecimientos la variación media geométrica de todas las variedades del mismo producto que sí tienen información de precios.

c) Precios faltantes para todos los establecimientos y todas las variedades del producto: se imputa la variación media geométrica de todos los productos del nivel superior de la misma agregación del producto.

d) Para los niveles superiores: se aplica lo indicado en el literal c.

e) Precios faltantes para los productos estacionales: se recolectan solo en los períodos para los cuales están disponible; en los meses restantes se mantienen los precios constantes.

f) En caso de que no sea posible imputar por el precio del agregado más cercano en el mismo nivel o niveles superiores, se procede a mantener el precio previo, es decir, los precios observados en el período anterior.

Para ilustrar el procedimiento a seguir para el cálculo del IPC se utilizará un diagrama de flujo elaborado por Eurostat (véase el diagrama 1).

Véanse las recomendaciones internacionales en Organización Internacional del Trabajo (OIT) y otros, Consumer Price Index Manual Concepts and Methods, 2020 [en línea] https://www.imf.org/en/Data/Statistics/cpi-manual, documento aprobado en el $51^{\circ}$ período de sesiones de la Comisión de Estadística de las Naciones Unidas, y Unión Europea, Harmonised Index of Consumer Prices (HICP) Methodological Manual, Luxemburgo, 2018 [en línea] https://ec.europa.eu/eurostat/documents/3859598/9479325/KS-GQ-17-015-EN-N.pdf/d5e63427c588-479f-9b19-f4b4d698f2a2. 
Diagrama 1

Árbol de decisión de Eurostat

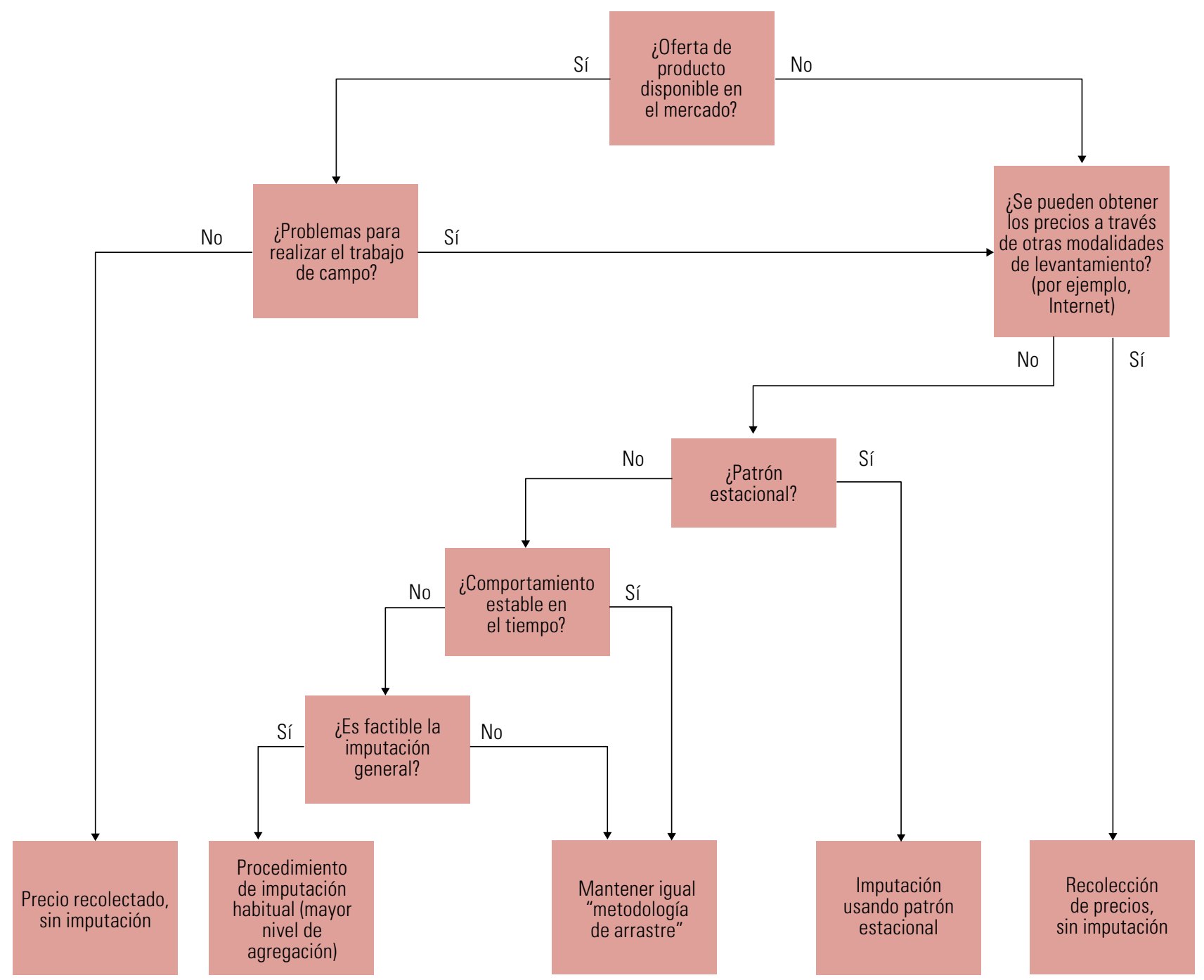

Fuente: Eurostat, presentación efectuada en la reunión extraordinaria del Grupo de Trabajo Intersecretarial sobre Estadísticas de Precios, 25 de marzo de 2020, inédito.

Además, a la aplicación de los respectivos algoritmos de cálculo para las imputaciones, en el ejercicio de marzo 2020 algunos países han realizado un análisis de los resultados para el mismo período febrero/marzo de 2018 y 2019, de manera tal de identificar patrones de comportamiento y así validar el resultado obtenido, en particular, para productos no estacionales.

\section{Desafíos observados en la implementación de las modalidades de recolección alternativas}

En la aplicación de las modalidades alternativas para la recolección de precios se encontraron algunas dificultades. Entre ellas pueden mencionarse la capacitación de los encuestadores de precios para la aplicación de estas modalidades y el establecimiento de un mecanismo de acceso a los informantes habituales para comunicarles el cambio en la modalidad de levantamiento y poder contar con su apoyo y disponibilidad en este nuevo escenario. Otra dificultad se refiere al cierre de fuentes información que no pudieron ser contactadas previamente para hacerles alguna solicitud. Todas estas situaciones deberán mejorarse, en lo posible, para dar continuidad a los levantamientos de precios de los próximos meses. 


\section{Difusión de las cifras en el nuevo escenario}

Dado que el nuevo escenario incide en la recolección de precios y su cobertura, es necesario poner al tanto de la situación a los usuarios respecto de la cobertura alcanzada, así como de las medidas tomadas para los levantamientos continuos de precios. De esta manera, es recomendable que a las cifras publicadas se añada una nota a pie de página o una nota sencilla pero explícita sobre la cobertura alcanzada incorporada en la difusión regular. Además, se recomienda elaborar una nota técnica con las medidas tomadas en cuanto al levantamiento y los procedimientos de imputación de los precios faltantes, así como también indicar el impacto de estas medidas. Por ejemplo, el Instituto Nacional de Estadística y Censos (INEC) del Ecuador elaboró una nota técnica sobre el tema para su publicación en marzo de $2020^{3}$. A continuación se presenta un extracto de la nota metodológica elaborada por el Instituto Nacional de Esatadísticas (INE) de Chile para el indicador de octubre.

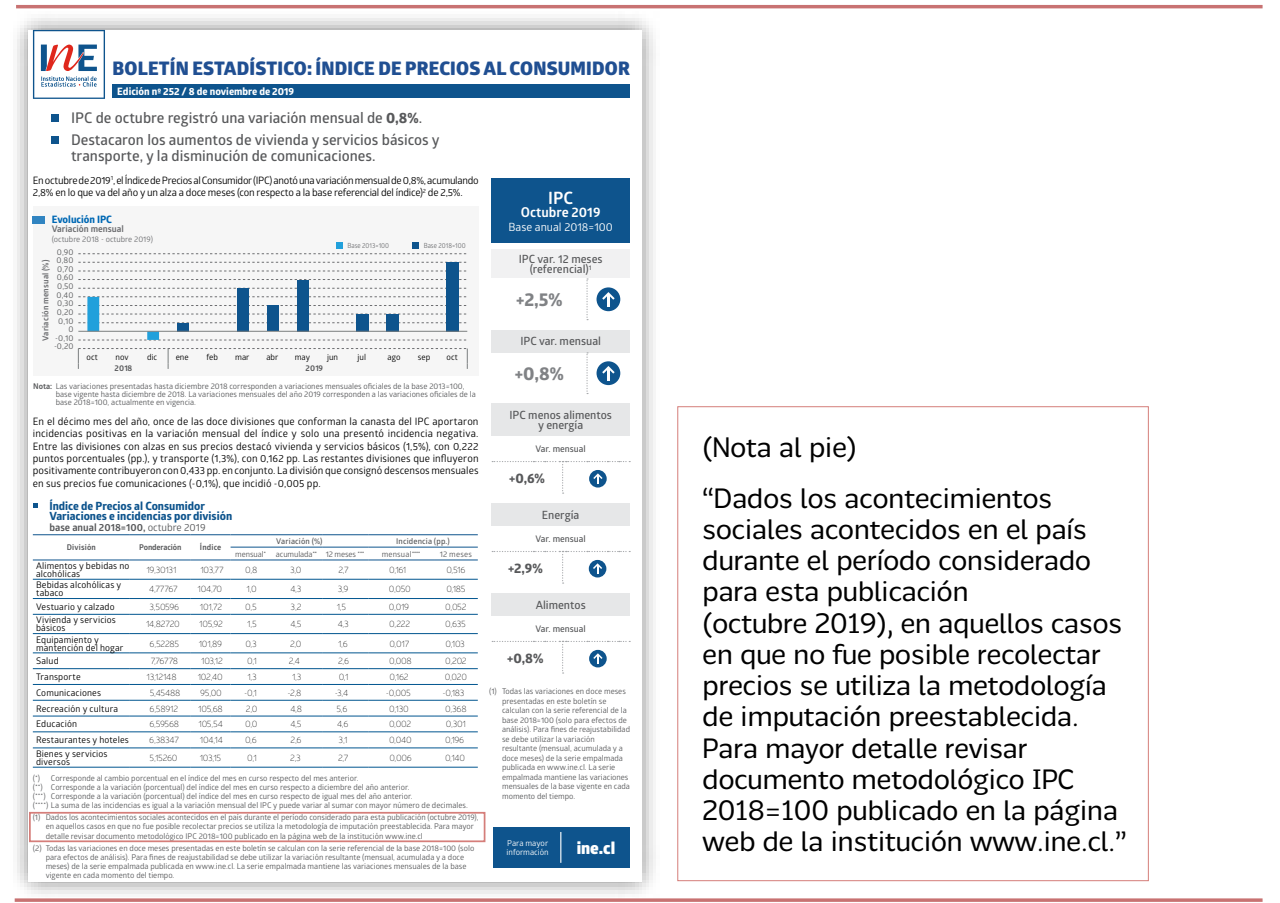

Fuente: Instituto Nacional de Estadísticas (INE) de Chile.

\section{E. Recomendaciones}

Ante esta situación de emergencia sanitaria y las dificultades señaladas, los objetivos principales son mantener una cobertura adecuada y garantizar la calidad de la información recolectada, así como también la calidad de los productos estadísticos resultantes de este proceso, en este caso el IPC.

Por ello, las instituciones de estadística productoras del IPC deberán continuar trabajando en la adaptación de los procesos de recopilación de sus datos (precios), procediendo con la recolección, el procesamiento y la difusión oportuna de sus resultados.

Para cumplir con este desafío es preciso crear rápidamente las capacidades necesarias en sus equipos, de manera de implementar la recolección remota utilizando tecnologías digitales en lugar de los métodos tradicionales basados en la consulta presencial cara a cara entre encuestador e informante. Uno de los factores clave en la selección de las nuevas modalidades es el tiempo de implementación para dar continuidad a los procesos de levantamiento y considerar, por ejemplo, capacitar al personal en el uso de nuevas metodologías, tecnologías y habilidades para obtener la mayor y mejor cobertura posible.

3 Véase [en línea] https://www.ecuadorencifras.gob.ec/documentos/web-inec/Inflacion/2020/Marzo-2020/ Nota_tecnica_cobertura_e_imputacion_03-2020-IPC.pdf. 
Por otra parte, es relevante hacer los ajustes necesarios para la incorporación de la información obtenida en los sistemas actuales. La captura de los precios de las mismas fuentes actuales utilizando mecanismos diferentes, ya sea mediante comunicación telefónica, correo electrónico o visita acotada, permitiría obtener los precios observados para las mismas fuentes existentes en las bases de precios del IPC. Sin embargo, la información recolectada a través de mecanismos digitales como captura en páginas web de los sitios de los informantes o a través de Internet, así como la disponibilidad de información de registro, podría tener como resultado información complementaria de las fuentes actuales, y por ello tendrá que ser incorporada en las bases de datos de precios, de forma diferenciada, de manera tal que sea posible tomar la decisión de volver a la modalidad de recolección presencial y poder excluir, si así se decide, esta información adicional una vez pasada la actual emergencia sanitaria.

La idea subyacente en este documento es que en la coyuntura actual los compiladores podrán introducir cierta flexibilidad en la recopilación de los precios, así como en su imputación, y se recomienda agotar estas instancias en lugar de recurrir a la ampliación de la muestra con fuentes nuevas. Si hay locales cerrados, no se recomienda realizar reemplazos de estos.

Por otra parte, considerando que la actual contingencia pudiera extenderse en el tiempo, se deberá buscar la mejora en la implementación de la recolección, incorporando nuevos mecanismos de captura y realizando los análisis correspondientes, no solo en el mes de observación sino con una mirada de mediano y largo plazo, teniendo presente los períodos similares para los años previos, y así poder conocer la consistencia de los resultados de las imputaciones realizadas en los precios faltantes.

Finalmente, se sugiere que las instituciones que se ven enfrentadas a esta nueva realidad en la recolección de sus precios:

- Implementen nuevos mecanismos para identificar, contactar y autenticar a los informantes y comunicarse con ellos.

- Implementen mecanismos para apoyar y supervisar los flujos de trabajo de las consultas telefónicas, consultas por correo electrónico y consultas por redes sociales de las fuentes informantes.

- Procuren o desarrollen sistemas de tecnologías de la información y las comunicaciones para apoyar las consultas telefónicas, por correo electrónico y por redes sociales, ya sea asistidas por computadora o en portales para la captura en línea, de ser posible, de modo tal que estén disponibles lo antes posible para su uso por parte de los analistas del IPC.

- Implementen mecanismos para apoyar a los analistas en la captura en línea de la información, su verificación y validación para incorporarla en las bases de datos de precios

- Garanticen un acceso remoto seguro a los sistemas informáticos y un intercambio de datos seguro con los analistas y encargados de los procesamientos y cálculos del IPC.

- Creen nuevas variables para la incorporación de la geocodificación automática de las respuestas a las consultas realizadas y la supervisión del proceso realizado

- Aprovechen el conocimiento adquirido en esta situación coyuntural para reflexionar respecto de la introducción de nuevos mecanismos para la recolección de información a través de las plataformas tecnológicas en el corto plazo.

\section{F. Aspectos de representatividad del indicador}

Tomando en consideración todos estos desafíos, se hace necesario plantear objetivos de cumplimiento para que el cálculo del IPC tenga la calidad necesaria para ser considerado un resultado adecuado como instrumento estadístico de seguimiento de la inflación y el monitoreo de los precios del consumo de los hogares. Por ello, teniendo presente que habitualmente existe un porcentaje de imputación de precios faltantes por no observación, en la situación actual se esperaría que para realizar el cálculo del IPC fuera suficiente contar con al menos un 50\% de la información a ser observada en el mes. 


\section{Anexo 1}

Ejemplos de procedimientos de imputaciones

A. Imputación de variedad

\begin{tabular}{|c|c|c|c|c|c|c|c|c|c|c|c|}
\hline $\begin{array}{l}\text { Código } \\
\text { de ítem }\end{array}$ & Nivel & $\begin{array}{l}\text { Código de } \\
\text { establecimiento }\end{array}$ & Glosa & Especificación & $\begin{array}{c}\text { Precio } \\
(\mathrm{t}-1)\end{array}$ & $\begin{array}{l}\text { Precio } \\
(\mathrm{t})\end{array}$ & $\begin{array}{c}\text { Relativo } \\
(\mathrm{t} / \mathrm{t}-1)\end{array}$ & $\begin{array}{c}\text { Media } \\
\text { geometrica }\end{array}$ & $\begin{array}{c}\text { Índice de } \\
\text { variedad- } \\
\text { establecimiento }\end{array}$ & $\begin{array}{l}\text { Indice de } \\
\text { variedad }\end{array}$ & \multirow{6}{*}{$\begin{array}{c}\text { Variación de } \\
\text { los precios } \\
\text { observados } \\
\text { que se aplica } \\
\text { a los precios } \\
\text { faltantes }\end{array}$} \\
\hline 02.13 & Clase & & Cerveza & & & & & & & & \\
\hline 02.1 .3 .0 & Subclase & & Cerveza & & & & & & & & \\
\hline 02.1.3.0.1 & Producto & & Cerveza nacional & & & & & & & & \\
\hline 02.1.3.0.1.1 & Variedad & & $\begin{array}{l}\text { Cerveza nacional } \\
\text { clara }\end{array}$ & & & & & & & 101,09 & \\
\hline 02.1 .3 .0 .1 .1 & Varied-Estab & $101-1$ & Supermercado 1 & Bavaria, botella & 1090 & 1100 & 100,92 & & 100,92 & & \\
\hline 02.1 .3 .0 .1 .1 & Varied-Estab & $101-2$ & Supermercado 2 & $\begin{array}{l}\text { Pilsen } 6.0 \text {, } \\
\text { botella }\end{array}$ & 770 & 775 & 100,65 & 1 & 100,65 & & \\
\hline 02.1 .3 .0 .1 .1 & Varied-Estab & $101-3$ & Supermercado 3 & $\begin{array}{l}\text { Imperial, lata de } \\
710 \mathrm{ml}\end{array}$ & 1180 & 1200 & 101,69 & 101,09 & 101,69 & & \\
\hline 02.1 .3 .0 .1 .1 & Varied-Estab & $103-1$ & Botillería 1 & $\begin{array}{l}\text { Pilsen 6.0, } \\
\text { botella }\end{array}$ & 818 & 827 & 4 & & 101,09 & & \\
\hline 02.1 .3 .0 .1 .1 & Varied-Estab & $103-2$ & Botillería 2 & Imperial, botella & 1190 & 1203 & & & 101,09 & & \\
\hline 02.1 .3 .0 .1 .1 & Varied-Estab & $103-3$ & Botillería 3 & Bavaria, botella & 1030 & 1041 & & & 101,09 & & \\
\hline 02.1.3.0.1.2 & Variedad & & $\begin{array}{l}\text { Cerveza nacional } \\
\text { oscura }\end{array}$ & & & & & & & & \\
\hline 02.1 .3 .0 .2 & Producto & & $\begin{array}{l}\text { Cerveza } \\
\text { importada }\end{array}$ & & & & & & & & $\begin{array}{l}\text { Precio } \\
\text { imputado }\end{array}$ \\
\hline 02.1 .3 .0 .2 .1 & Variedad & & $\begin{array}{l}\text { Cerveza } \\
\text { importada clara }\end{array}$ & & & & & & & & $\begin{array}{l}\text { usando la } \\
\text { variación de }\end{array}$ \\
\hline 02.1 .3 .0 .2 .2 & Variedad & & $\begin{array}{l}\text { Cerveza } \\
\text { importada } \\
\text { oscura }\end{array}$ & & & & & & & & observados \\
\hline
\end{tabular}

En este ejercicio se ejemplifica el procedimiento de imputación cuando falta una parte de los precios para algunos registros de la variedad, como en el caso de los precios de "Cerveza nacional" para la tipología de establecimiento "botillería".

B. Imputación de producto

\begin{tabular}{|c|c|c|c|c|c|c|c|c|c|c|}
\hline $\begin{array}{l}\text { Código } \\
\text { de ítem }\end{array}$ & Nivel & $\begin{array}{l}\text { Código de } \\
\text { establecimiento }\end{array}$ & Glosa & Especificación & $\begin{array}{l}\text { Precio } \\
(\mathrm{t}-1)\end{array}$ & $\begin{array}{l}\text { Precio } \\
\text { (t) }\end{array}$ & $\begin{array}{c}\text { Relativo } \\
(\mathrm{t} / \mathrm{t}-1)\end{array}$ & $\begin{array}{c}\text { Media } \\
\text { geométrica }\end{array}$ & $\begin{array}{l}\text { Indice de } \\
\text { producto }\end{array}$ & \\
\hline 09.3 .2 .2 & Subclase & & $\begin{array}{l}\text { Productos para } \\
\text { mascotas y otros } \\
\text { animales del hogar }\end{array}$ & & & & & & & $\begin{array}{l}\text { Precio } \\
\text { imputado } \\
\text { usando la }\end{array}$ \\
\hline 09.3.2.2.1 & Producto & & Servicios veterinarios & & & & & & 100,85 & variación de \\
\hline \multirow[t]{3}{*}{ 09.3.2.2.1.1 } & Variedad & & Servicios de vacunación & & & 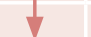 & & 100,85 & & \\
\hline & Varied-Estab & $201-1$ & Vacuna antirrábica & $\begin{array}{l}\text { Perro menor } \\
\text { de } 1 \text { año }\end{array}$ & 9900 & 9984 & 100,85 & & & \\
\hline & Varied-Estab & $201-2$ & Vacuna antirrábica & $\begin{array}{l}\text { Perro menor } \\
\text { de } 1 \text { año }\end{array}$ & 10800 & 10891 & 100,85 & & & \\
\hline \multirow[t]{3}{*}{09.3 .2 .2 .1 .2} & Variedad & & $\begin{array}{l}\text { Servicios de } \\
\text { desparasitación }\end{array}$ & & & & & 100,85 & & \\
\hline & Varied-Estab & $201-1$ & $\begin{array}{l}\text { Consulta con } \\
\text { desparasitación }\end{array}$ & $\begin{array}{l}\text { Perro de } \\
3 \text { meses }\end{array}$ & 5500 & 5547 & 100,85 & & & \\
\hline & Varied-Estab & $201-3$ & $\begin{array}{l}\text { Consulta con } \\
\text { desparasitación }\end{array}$ & $\begin{array}{l}\text { Perro de } \\
3 \text { meses }\end{array}$ & 6500 & 6555 & 100,85 & & & \\
\hline 09.3 .2 .2 .2 & Producto & & $\begin{array}{l}\text { Alimentos para } \\
\text { mascotas }\end{array}$ & & & & & & 100,85 & $\begin{array}{c}\text { calculado con } \\
\text { la información } \\
\text { observada }\end{array}$ \\
\hline \multirow[t]{4}{*}{ 09.3.2.2.2.1 } & Variedad & & Alimentos para perros & & & & & 101,12 & & \\
\hline & Varied-Estab & $101-1$ & $\begin{array}{l}\text { Alimento seco } \\
\text { para perro adulto }\end{array}$ & $\begin{array}{l}\text { Pro Pet, } \\
1.500 \text { grs }\end{array}$ & 4250 & 4300 & 101,18 & & & \\
\hline & Varied-Estab & $101-2$ & $\begin{array}{l}\text { Alimento seco } \\
\text { para perro adulto }\end{array}$ & $\begin{array}{l}\text { Super Perro, } \\
2.000 \text { grs }\end{array}$ & 6900 & 7000 & 101,45 & & & \\
\hline & Varied-Estab & $104-1$ & $\begin{array}{l}\text { Alimento seco } \\
\text { para perro adulto }\end{array}$ & $\begin{array}{l}\text { Beneful, } 1.590 \\
\text { grs }\end{array}$ & 10950 & 11030 & 100,73 & & & \\
\hline \multirow[t]{4}{*}{ 09.3.2.2.2.2 } & Variedad & & Alimentos para gatos & & & & & 100,58 & & \\
\hline & Varied-Estab & $101-1$ & $\begin{array}{l}\text { Alimento seco } \\
\text { para gatos }\end{array}$ & $\begin{array}{l}\text { Cat Chow, } \\
1.500 \text { grs }\end{array}$ & 5400 & 5430 & 100,56 & & & \\
\hline & Varied-Estab & $101-2$ & $\begin{array}{l}\text { Alimento seco } \\
\text { para gatos }\end{array}$ & $\begin{array}{l}\text { Whiskas, } \\
1.500 \text { grs }\end{array}$ & 3880 & 3890 & 100,26 & & & \\
\hline & Varied-Estab & $104-2$ & $\begin{array}{l}\text { Alimento seco } \\
\text { para gatos }\end{array}$ & $\begin{array}{l}\text { Royal Canin, } \\
2.000 \text { grs }\end{array}$ & 16370 & 16520 & 100,92 & & & \\
\hline
\end{tabular}

En este ejemplo se observa la falta de precios para un producto "Servicios de veterinaria", al cual se le imputa la variación de otro producto "Alimentos para mascotas". 
C. Imputación de subclase

\begin{tabular}{|c|c|c|c|c|c|c|c|c|c|c|}
\hline $\begin{array}{l}\text { Código } \\
\text { de ítem }\end{array}$ & Nivel & $\begin{array}{l}\text { Ponderación } \\
\text { de producto }\end{array}$ & Glosa & Especificación & $\begin{array}{l}\text { Indice } \\
(\mathrm{t}-1)\end{array}$ & $\begin{array}{l}\text { Relativo } \\
\text { (t) }\end{array}$ & $\begin{array}{l}\text { Indice de } \\
\text { variedad }\end{array}$ & $\begin{array}{l}\text { Indice de } \\
\text { producto }\end{array}$ & $\begin{array}{l}\text { Indice de } \\
\text { subclase }\end{array}$ & \\
\hline 05.1 .1 & Clase & & $\begin{array}{l}\text { Muebles, accesorios } \\
\text { y alfombras sueltas }\end{array}$ & & & & & & & $\begin{array}{c}\text { Indice } \\
\text { imputado }\end{array}$ \\
\hline 05.1.1.1 & Subclase & & Muebles & & 104,51 & & & & 104,53 & variación \\
\hline 05.1.1.1.1 & Producto & 0,35 & Comedor & & 105,99 & 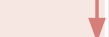 & & 106,01 & & calculada del \\
\hline 05.1.1.1.1. & Variedad & & Comedor & 6 personas & 106,03 & 100,01 & 106,04 & & & $\begin{array}{l}\text { mes }(t) \text { con } \\
\text { respecto a }(t-1)\end{array}$ \\
\hline 05.1 .1 .1 .1 .2 & Variedad & & Comedor & 4 personas & 105,96 & 100,01 & 105,97 & & & \\
\hline 05.1.1.1.2 & Producto & 0,27 & Camas & & 102,59 & & & 102,61 & & \\
\hline 05.1 .1 .1 .2 .1 & Variedad & & Cama & Individual & 104,59 & 100,01 & 104,60 & & & \\
\hline 05.1 .1 .1 .2 .2 & Variedad & & Cama & Matrimonial & 100,64 & 100,01 & 100,65 & & & Índice \\
\hline 05.1.1.4 & Subclase & & Accesorios y alfombras & & 105,97 & & & & 105,98 & $\begin{array}{l}\text { calculado con } \\
\text { la información }\end{array}$ \\
\hline 05.1.1.4.1 & Producto & 0,10 & Alfombra & & 108,78 & & & 109,16 & & observada \\
\hline 05.1.1.4.1.1 & Variedad & & Alfombra & Circular & 106,86 & 100,50 & 107,39 & & & \\
\hline 05.1 .1 .4 .1 .2 & Variedad & & Alfombra & Rectangular & 110,73 & 100,20 & 110,95 & & & \\
\hline $05 \cdot 1 \cdot 1.4 .2$ & Producto & 0,07 & Floreros & & 101,95 & & & 101,44 & & \\
\hline 05.1.1.4.2.1 & Variedad & & Florero & Cristal & 102,30 & 100,10 & 102,40 & & & \\
\hline 05.1 .1 .4 .2 .2 & Variedad & & Florero & Cerámica & 101,60 & 98,90 & 100,48 & & & \\
\hline
\end{tabular}

En este ejercicio se muestra la imputación a nivel de subclase usando la información de la otra subclase observada.

Fuente: Comisión Económica para América Latina y el Caribe (CEPAL).

Este documento es parte de un conjunto de informes elaborados por la Comisión Económica para América Latina y el Caribe (CEPAL) sobre la evolución y los efectos de la pandemia del COVID-19 en América Latina y el Caribe. Fue preparado por la División de Estadísticas, dirigida por Rolando Ocampo, bajo la coordinación general de Alicia Bárcena, Secretaria Ejecutiva de la CEPAL.

Copyright (C) Naciones Unidas, 2020 